\title{
Turbulent Forced Convection of Radiative Gas Flow in a Duct with Separation
}

\author{
Amir Asghari, Seyyed Abdolreza Gandjalikhan Nassab \\ Mechanical Engineering Department, School of Engineering, Shahid Bahonar University, Kerman, Iran \\ Email: amir.asghari.62@gmail.com, Ganj110@mail.uk.ac.ir
}

Received May 9, 2013; revised June 9, 2013; accepted June 17, 2013

Copyright (C) 2013 Amir Asghari, Seyyed Abdolreza Gandjalikhan Nassab. This is an open access article distributed under the Creative Commons Attribution License, which permits unrestricted use, distribution, and reproduction in any medium, provided the original work is properly cited.

\begin{abstract}
In the present work, a numerical solution is described for turbulent forced convection flow of an absorbing, emitting, scattering and gray fluid over a two-dimensional backward facing step in a horizontal duct. The AKN low-Reynolds-number model is employed to predict turbulent flows with separation and heat transfer, while the radiation part of the problem is modeled by the discrete ordinate method (DOM). Discretized forms of the governing equations for fluid flow are obtained by finite volume approach and solved using SIMPLE algorithm. Results are presented for the distributions of Nusselt numbers as a function of the controlling parameters like radiation-conduction parameter (RC) and optical thickness.
\end{abstract}

Keywords: Backward Facing Step; Turbulent Forced Convection Flow; Radiation Heat Transfer; Discrete Ordinate Method

\section{Introduction}

Flow in ducts with combined convection, conduction, and radiation in participating media occurs in many engineering applications, such as solar collectors, combustion chambers, industrial furnaces, gas turbine blades and so on. An extensively known geometry is the backward facing step (BFS) flow that has the most features of separated flows. Although the geometry of BFS flow is very simple, many aspects of the heat transfer and fluid flow structure remain incompletely explained.

Several investigations like [1,2] have been done over BFS convection flow in a duct, both about laminar and turbulent regimes. Some important measurements in turbulent convection flow downstream of a BFS were done by Adams et al. [3] and Vogel and Eaton [4]. Abe et al. [5,6] found a quite successfully numerical turbulent model, and tested their codes with these experimental results. The present research work was carried out to add radiation effect to this problem with considering a participating media. Similar research studies have been done for fluid flows with simple geometries, such as pipe flow and flow between parallel plates $[7,8]$. To the best of author's knowledge, the forced convection turbulent flow over BFS has not been studied using AKN low Reynolds turbulent model in flow calculation with DOM in solving radiation problem.

\section{Problem Statement}

Two-dimensional turbulent forced convection flow in a rectangular duct with a BFS is numerically simulated. A schematic of the computational domain is shown in Figure 1. The channel height, $H$, is $0.19 \mathrm{~m}$, and the step height, $h$, is $0.038 \mathrm{~m}$, which is considered as the characteristic length in the computation. The upstream and downstream lengths of the step are 0.076 and $0.760 \mathrm{~m}$, respectively, which is corresponds to $-2 \leq x / h \leq 20$, in the computational domain (Figure 1).

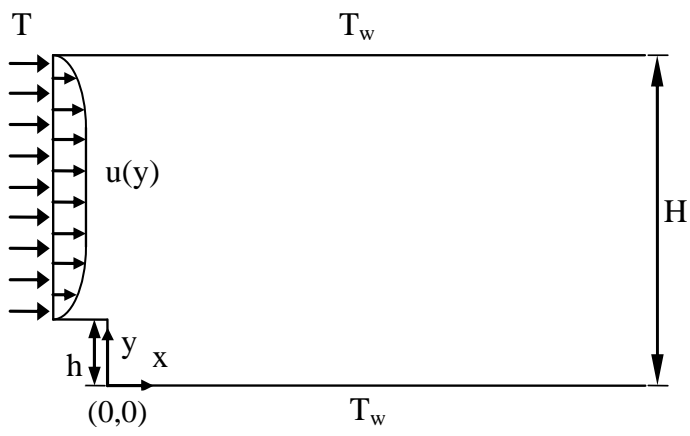

Figure 1. Sketch of problem geometry. 
In the test case related to numerical validation, the fluid physical properties are treated as constants and evaluated for air at the inlet temperature of $T_{0}=20^{\circ} \mathrm{C}$ (i.e. density $(\rho)$ is $1.205 \mathrm{~kg} / \mathrm{m}^{3}$, molecular dynamic viscosity $(\mu)$ is $1.782 \times 10^{-5} \mathrm{~kg} / \mathrm{ms}$, specific heat $\left(C_{p}\right)$ is 1005 $\mathrm{J} /\left(\mathrm{kg}^{\circ} \mathrm{C}\right)$ and Prandtl number $(P r)$ is 0.71$)$. The channel expansion ratio is 1.25 , with a Reynolds number of 28,000 based on the centerline velocity at the inlet section $\left(u_{0}=10.86 \mathrm{~m} / \mathrm{s}\right)$ and step height.

\subsection{Basic Equations}

For predicting turbulent flow and heat transfer in separating and reattaching flows, quite successfully AKN model that introduced by Abe et al. [5,6], was selected for this study.

The governing equations for BFS flow, which are considered to be 2-D, steady, incompressible and turbulent are the equation of continuity, the Reynolds averaged Navier-Stokes equation, the equations of the turbulent kinetic energy $k$ for the velocity field and its dissipation rate $\varepsilon$, the energy equation, and the equations of the turbulent kinetic energy $t^{2}$ for the thermal field and its dissipation rate $\varepsilon_{t}$ that can be written as follows:

Continuity:

$$
\frac{\partial u_{i}}{\partial x_{i}}=0
$$

Two-equation model for velocity field:

$$
\begin{gathered}
\frac{D u_{i}}{D \hat{t}}=-\frac{1}{\rho} \frac{\partial p}{\partial x_{i}}+\frac{\partial}{\partial x_{j}}\left\{v\left(\frac{\partial u_{i}}{\partial x_{j}}+\frac{\partial u_{j}}{\partial x_{i}}\right)-\overline{u_{i}^{\prime} u_{j}^{\prime}}\right\} \\
\frac{D k}{D \hat{t}}=\frac{\partial}{\partial x_{j}}\left\{\left(v+\frac{v_{t}}{\sigma_{k}}\right) \frac{\partial k}{\partial x_{j}}\right\}-\overline{u_{i}^{\prime} u_{j}^{\prime}} \frac{\partial u_{i}}{\partial x_{j}}-\varepsilon \\
\frac{D \varepsilon}{D \hat{t}}=\frac{\partial}{\partial x_{j}}\left\{\left(v+\frac{v_{t}}{\sigma_{\varepsilon}}\right) \frac{\partial \varepsilon}{\partial x_{j}}\right\}-C_{\varepsilon 1} \frac{\varepsilon}{k} \overline{u_{i}^{\prime} u_{j}^{\prime}} \frac{\partial u_{i}}{\partial x_{j}}-C_{\varepsilon 2} f_{\varepsilon} \frac{\varepsilon^{2}}{k}
\end{gathered}
$$

with

$$
\overline{-u_{i}^{\prime} u_{j}^{\prime}}=v_{t}\left(\frac{\partial u_{i}}{\partial x_{j}}+\frac{\partial u_{j}}{\partial x_{i}}\right)-\frac{2}{3} k \delta_{i j}
$$

Two-equation model for thermal field (note that molecular viscosity is negligible):

$$
\begin{aligned}
& \frac{D T}{D \hat{t}}=\frac{\partial}{\partial x_{j}}\left(\alpha \frac{\partial T}{\partial x_{j}}-\overline{u_{j}^{\prime} t}\right)-\frac{\nabla \cdot \boldsymbol{q}_{r}}{\rho C_{p}} \\
& \frac{D \overline{t^{2}}}{D \hat{t}}=\frac{\partial}{\partial x_{j}}\left\{\left(\alpha+\frac{\alpha_{t}}{\sigma_{h}}\right) \frac{\partial \overline{t^{2}}}{\partial x_{j}}\right\}-2 \overline{u_{j}^{\prime} t} \frac{\partial T}{\partial x_{j}}-2 \varepsilon_{t}
\end{aligned}
$$

$$
\begin{aligned}
& \frac{D \varepsilon_{t}}{D \hat{t}}=\frac{\partial}{\partial x_{j}}\left\{\left(\alpha+\frac{\alpha_{t}}{\sigma_{\phi}}\right) \frac{\partial \varepsilon_{t}}{\partial x_{j}}\right\}-C_{P 1} f_{P 1} \frac{\varepsilon_{t}}{\overline{t^{2}}} \overline{u_{j}^{\prime} t} \frac{\partial T}{\partial x_{j}} \\
& -C_{P 2} f_{P 2} \frac{\varepsilon_{t}}{k} \overline{u_{i}^{\prime} u_{j}^{\prime}} \frac{\partial u_{i}}{\partial x_{j}}-C_{D 1} f_{D 1} \frac{\varepsilon_{t}^{2}}{\overline{t^{2}}}-C_{D 2} f_{D 2} \frac{\varepsilon \varepsilon_{t}}{k}
\end{aligned}
$$

with

$$
\overline{-u_{j}^{\prime} t}=\alpha_{t} \frac{\partial T}{\partial x_{j}}
$$

where

$$
\begin{gathered}
v_{t}=C_{\mu} f_{\mu} k^{2} / \varepsilon \\
\alpha_{t}=C_{\lambda} f_{\lambda} k^{2} / \varepsilon \\
f_{\mu}=\left\{1-\exp \left(-\frac{y^{*}}{14}\right)\right\}^{2}\left[1+\frac{5}{R_{t}^{3 / 4}} \exp \left\{-\left(\frac{R_{t}}{200}\right)^{2}\right\}\right] \\
f_{\varepsilon}=\left\{1-\exp \left(-\frac{y^{*}}{3.1}\right)\right\}^{2}\left[1-0.3 \exp \left\{-\left(\frac{R_{t}}{6.5}\right)^{2}\right\}\right] \\
f_{\lambda}=\left\{\left(\frac{2 R}{C_{m}+R}\right)+3 \frac{(v \varepsilon)^{3 / 4}}{k^{3 / 2}} \frac{(2 R)^{1 / 2}}{P r} f_{d}\right\} \\
\times\left\{1-\exp \left(-\frac{y^{*}}{14}\right)\right\}\left\{1-\exp \left(-\frac{P r^{1 / 2} y^{*}}{14}\right)\right\} \\
f_{d}=\exp \left\{-\left(\frac{R_{t}}{200}\right)^{2}\right\} \\
f_{D 1}=f_{P 1}=\left\{1-\exp \left(-\frac{y^{*}}{A_{D 1}}\right)\right\}^{2} ; f_{P 2}=1.0 \\
R_{t}=k^{2} / v \varepsilon \\
f_{D 2}=\frac{C_{\varepsilon 2} f_{2}-1}{C_{D 2}}\left\{1-\exp \left(-\frac{y^{*}}{A_{D 2}}\right)\right\}^{2} \\
f_{2}=1-0.3 \exp \left\{-\left(\frac{R_{t}}{6.5}\right)^{2}\right\}
\end{gathered}
$$

In the above equations, $\overline{u_{i}^{\prime} u_{j}^{\prime}}$ is the Reynolds stress component and $\overline{u_{j}^{\prime} t}$ is the turbulent heat flux. Also, the constants parameters in the governing equations are given in Table 1.

At the inlet duct section, the fluid flow consists a uniform temperature profile ( $\left.T_{0}=600 \mathrm{~K}\right)$. Also, the walls considered isotherm with temperature of $750 \mathrm{~K}$. 
Table 1. Model constants appearing in the governing equations.

\begin{tabular}{ccccccccccccccc}
\hline$C_{\mu}$ & $\sigma_{k}$ & $\sigma_{\varepsilon}$ & $C_{m}$ & $C_{\varepsilon 1}$ & $C_{\varepsilon 2}$ & $C_{\lambda}$ & $C_{D 1}$ & $C_{D 2}$ & $\sigma_{h}$ & $\sigma_{\phi}$ & $C_{P 1}$ & $C_{P 2}$ & $A_{D 1}$ & $A_{D 2}$ \\
\hline 0.09 & 1.4 & 1.4 & 0.5 & 1.5 & 1.9 & 0.1 & 2 & 0.9 & 1.6 & 1.6 & 1.9 & 0.6 & 1 & 5.7 \\
\hline
\end{tabular}

\subsection{Gas Radiation Modeling}

In presence of participating media with radiation effect, besides the convective and conductive terms in the energy equation, the radiative term $\nabla \cdot \boldsymbol{q}_{r}$ is also exist that can be calculated as [9]:

$$
\nabla \cdot \boldsymbol{q}_{r}=\sigma_{a}\left(4 \pi I_{b}(\boldsymbol{r})-\int_{4 \pi} I(\boldsymbol{r}, \boldsymbol{s}) \mathrm{d} \Omega\right)
$$

where, $\boldsymbol{r}$ and $\boldsymbol{s}$ are position and direction of the radiation intensity $I(\boldsymbol{r}, \boldsymbol{s})$. To obtain the radiation intensity field and then the term $\nabla \cdot \boldsymbol{q}_{r}$, we should solve the radiative transfer equation (RTE) firstly, that for an absorbing, emitting and scattering gray medium can be written as:

$$
\begin{aligned}
& (\boldsymbol{s} \cdot \nabla) I(\boldsymbol{r}, \boldsymbol{s}) \\
& =-\beta I(\boldsymbol{r}, \boldsymbol{s})+\sigma_{a} I_{b}(\boldsymbol{r})+\frac{\sigma_{s}}{4 \pi} \int_{4 \pi} I(\boldsymbol{r}, \boldsymbol{s}) \varphi\left(\boldsymbol{s}, \boldsymbol{s}^{\prime}\right) \mathrm{d} \Omega^{\prime}
\end{aligned}
$$

in which $\boldsymbol{s}^{\prime}$ is incoming and $\boldsymbol{s}$ is scattered directions and $\varphi\left(s, s^{\prime}\right)$ is the scattering phase function which is equal to unity for isotropic scattering media. The numerical procedure in solving RTE (that is the DOM) was given in detail by the second author in his previous work [10]. By this method, heat flux may also be determined from surface energy balance, as:

$$
\boldsymbol{q} \cdot \boldsymbol{n}\left(\boldsymbol{r}_{w}\right) \cong \varepsilon_{w}\left(\pi I_{b}\left(\boldsymbol{r}_{w}\right)-\sum_{\boldsymbol{n} \cdot s_{i}<0} w_{i} I_{i}\left(\boldsymbol{r}_{w}\right)\left|\boldsymbol{n} \cdot \boldsymbol{s}_{i}\right|\right)
$$

The boundary conditions for the radiative problem are treated as diffusely walls with constant emissivity of $\varepsilon_{w}=0.8$. In addition, the inlet and outlet sections are considered as pseudo-black walls at their temperatures equal to fluid temperature in inlet and outlet sections, respectively [11].

The local total Nusselt number along the duct walls is defined as $N u_{t}=q_{t} h / \kappa\left(T_{w}-T_{b}\right)$ where $q_{t}$ represents the sum of convective and radiative heat fluxes such that $q_{t}=q_{c}+q_{r}=-\kappa(\partial T / \partial y)+q_{r}$. Therefore, the function $N u_{t}$ is the sum of local convective Nusselt number, $N u_{c}$, and local radiative Nusselt number, $N u_{r}$.

\subsection{Non-Dimensional Forms of the Governing Equations}

In the numerical solution of governing equations, the following dimensionless parameters are used to obtain the non-dimensional forms of the equations:

$$
\begin{aligned}
& x_{i}^{*}=\frac{x_{i}}{h}, u_{i}^{*}=\frac{u_{i}}{u_{0}}, p^{*}=\frac{p}{\rho u_{0}^{2}}, \hat{t}^{*}=\frac{\hat{t}}{h / u_{0}}, \\
& k^{*}=\frac{k}{u_{0}^{2}}, \varepsilon^{*}=\frac{\varepsilon h}{u_{0}^{3}}, T^{*}=\frac{T-T_{0}}{T_{w}-T_{0}}, \operatorname{Pr}=\frac{v}{\alpha} \text {, } \\
& \overline{t^{* 2}}=\frac{\overline{t^{2}}}{\left(T_{w}-T_{0}\right)^{2}}, \varepsilon_{t}^{*}=\frac{\varepsilon_{t} h / u_{0}}{\left(T_{w}-T_{0}\right)^{2}}, y_{n}^{*}=\frac{y_{n}}{h} \text {, } \\
& \overline{u_{i}^{* *} u_{j}^{\prime *}}=\frac{\overline{u_{i}^{\prime} u_{j}^{\prime}}}{u_{0} v / h}, \overline{u_{j}^{\prime *} t^{*}}=\frac{\overline{u_{j}^{\prime} t}}{\alpha\left(T_{w}-T_{0}\right) / h}, \\
& I^{*}=\frac{I}{\sigma T_{w}^{4}}, \boldsymbol{q}_{r}^{*}=\frac{\boldsymbol{q}_{r}}{\sigma T_{w}^{4}}, v_{t}^{*}=\frac{v_{t}}{v}, \alpha_{t}^{*}=\frac{\alpha_{t}}{\alpha}
\end{aligned}
$$

For example, non-dimensional form of Equation (6) is:

$$
\begin{aligned}
& \frac{D T^{*}}{D \hat{t}^{*}}=\frac{1}{P e} \frac{\partial}{\partial x_{j}^{*}}\left(\frac{\partial T^{*}}{\partial x_{j}^{*}}-\overline{u_{j}^{\prime *} t^{*}}\right) \\
& -\frac{\tau(1-\omega) \operatorname{RC} \theta_{1} \theta_{2}}{P e}\left[\frac{4}{\theta_{2}^{4}}\left(\frac{T^{*}}{\theta_{1}}+1\right)^{4}-\sum_{i=1}^{n} I_{i}^{*} w_{i}\right]
\end{aligned}
$$

Two physical quantities of interest in heat transfer study are the mean bulk temperature and the convective and radiative Nusselt numbers which are defined by:

$$
\begin{gathered}
T_{b}^{*}=\frac{\int_{0}^{1} T^{*} u^{*} \mathrm{~d} y^{*}}{\int_{0}^{1} u^{*} \mathrm{~d} y^{*}} \\
N u_{t}=N u_{c}+N u_{r}=\left.\frac{-1}{T_{w}^{*}-T_{b}^{*}} \frac{\partial T^{*}}{\partial y^{*}}\right|_{y^{*}=0}+\frac{\mathrm{RC} \cdot \theta_{1} \cdot \theta_{2}}{T_{w}^{*}-T_{b}^{*}} q_{r}^{*}
\end{gathered}
$$

\section{Numerical Procedure}

The governing equations are solved numerically by the CFD techniques to obtain the velocity and temperature fields. Discrete procedure utilizes the method of line-byline in conjunction with finite volumes that coded into a computer program in FORTRAN and solved by SIMPLE algorithm of Patankar and Spalding [12].

Based on the grid-independent study, several grid distributions were performed and the grids of $430(x) \times$ $280(y)$ downstream of the step were selected for the numerical analysis, while using denser mesh of $470(x) \times$ $330(y)$ resulted in less than $2 \%$ difference in the value of maximum total Nusselt number on the bottom wall (Table 2). Non-uniformly structured with highly concen- 
trated close to the wall surfaces and near the step corners and the reattachment zone, were used in order to ensure the accuracy of numerical solution.

Since, in the DOM, different numbers of discrete directions can be chosen during $S_{N}$ approximation, the results obtained by the $S_{4}, S_{8}$ and $S_{12}$ approximations were compared and there was a small difference, less than $2 \%$ error, between $S_{8}$ and $S_{12}$ approximations. Therefore, $S_{8}$ approximation has been used in subsequent calculations.

\section{Code Validation}

In order to validate the accuracy of convective heat transfer computations, a benchmark problem was selected. It deals to a turbulent convection flow over a BFS in a duct in which the bottom wall downstream of the step is supplied with a uniform heat flux $\left(q_{w}=270 \mathrm{~W} / \mathrm{m}^{2}\right)$, while other walls are treated as adiabatic surface. So predicted Stanton number profile on the bottom wall obtained by two-equation turbulence model compared with experimental data [4] and a numerical data [13] with assumption of constant turbulent Prandtl number, where exhibited in Figure 2. It can be seen that the two-equation turbulence model prediction is in better agreement with experiment.

It should be noted that as the radiating effect of the gas flow is neglected in that test case, the gas flow is considered non-participating media in the computation of Figure 2, where the validation of combined conductiveradiative heat transfer results was given by the second author in his previous work [10].

Table 2. Grid independence study, $\mathrm{RC}=25, \omega=0.5, \tau=15$.

\begin{tabular}{cccc}
\hline Grid size & $390 \times 230$ & $430 \times 280$ & $470 \times 330$ \\
$\left.N u_{t}\right|_{\max }$ & 71.98 & 77.51 & 78.87 \\
\hline
\end{tabular}

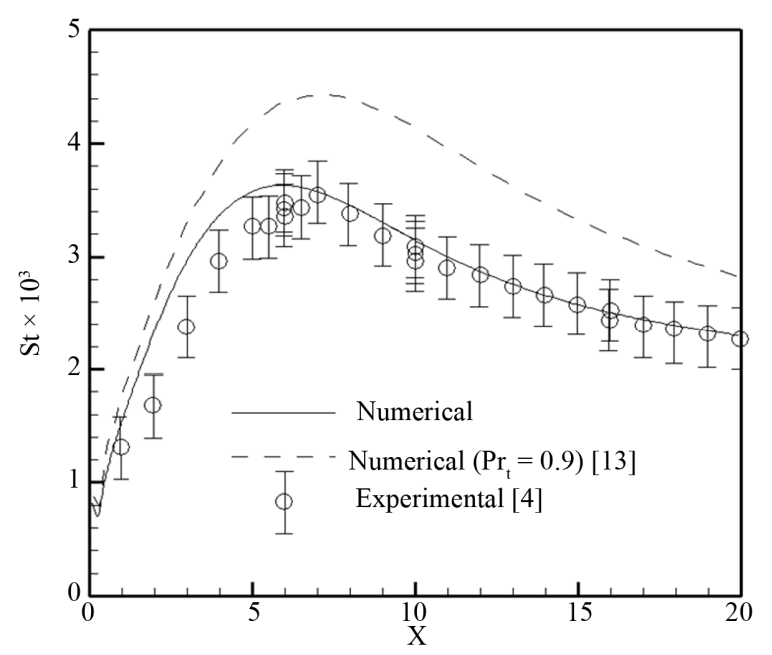

Figure 2. Comparison of the Stanton number with the experimental and theoretical results.

\section{Results and Discussions}

The numerical results are presented for a turbulent separated and reattached convection flow of a radiating gas over a 2-D BFS in a horizontal duct. The results represent how well the energy transfer from the wall to the gas as the fluid flow passes through the channel.

In order to show the variations of Nusselt numbers $\left(N u_{c, r, t}\right)$ along the bottom wall, Figure $\mathbf{3}$ is plotted with considering the effect of RC parameter, which shows the relative importance of the radiation mechanism compared with its conduction counterpart. Figure 3(a) illustrates the distribution of $N u_{r}$ along the bottom wall. It is seen that as the distance increases from the step corner,

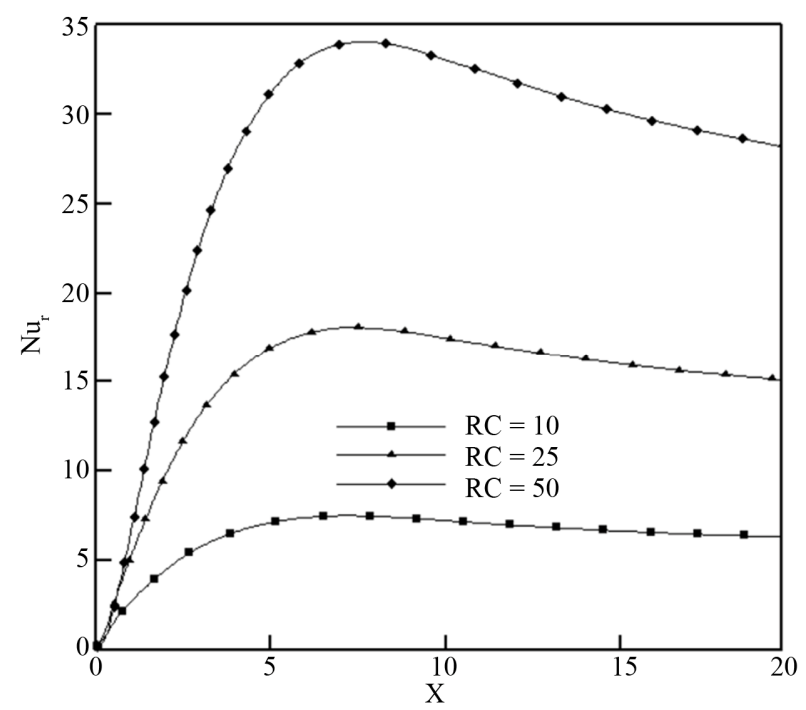

(a)

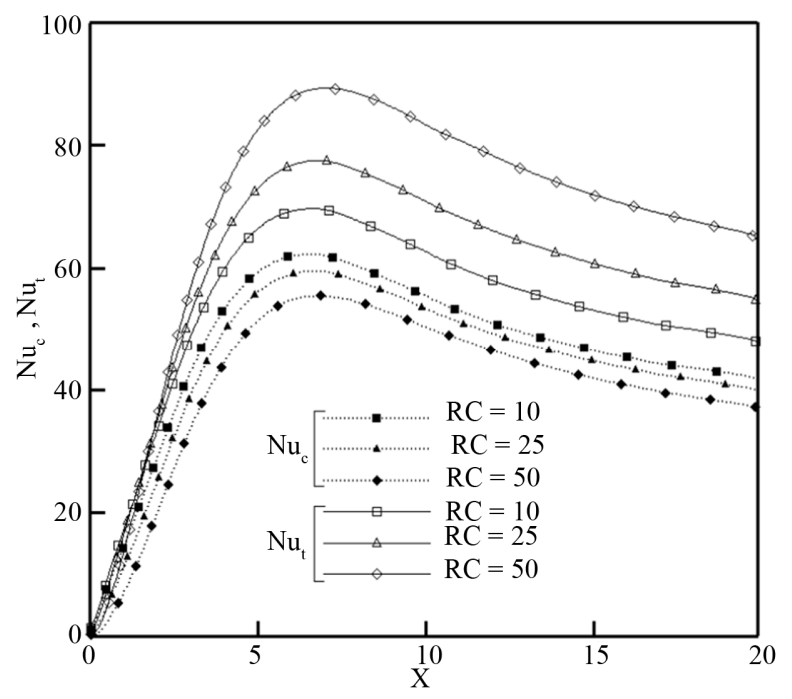

(b)

Figure 3. Effect of RC on the Nu distribution along the bottom wall, $\tau=15, \omega=0.5$ : (a) Radiative Nusselt number; (b) Convective and total Nusselt numbers. 
the value of radiative heat flux and consequently radiative Nusselt number increases sharply to its maximum value, which is due to a decreases in bottom wall incident radiative heat flux incoming from the stepped surface. After the maximum point, $N u_{r}$ decreases and approaches to a constant value as the distance continues to increase in the stream wise direction. Besides, Figure 3(a) shows that the $N u_{r}$ increases by increasing in RC, which is due to the increases in bottom wall's outgoing radiative heat flux.

The distributions of both conductive and total Nusselt numbers along the bottom wall are presented in Figure 3(b) at different values of the RC parameter. The variation of $N u_{c}$ shows an increasing trend in the recirculation zone after the step corner, such that the maximum value of $N u_{c}$ occurs at the reattachment point, after which the $N u_{c}$ approaches to a constant value far from the step location. Also, it is seen from Figure 3(b) that the $N u_{c}$ decreases by increasing in RC. This is due to this fact that under the effective presence of radiation heat transfer at high value of $\mathrm{RC}$, the temperature field inside the flow domain becomes more uniform. Consequently, the value of temperature gradient inside the flow domain decreases that causes a decrease in the value of convection coefficient on the bottom wall. The distribution of total Nusselt number is also shown in Figure 3(b). It is seen that $N u_{t}$ and $N u_{c}$ have similar trend but Figure 3(b) illustrates that $N u_{t}$ increases with increasing in RC. One can easily analyze this trend by considering the definition of $N u_{t}$, and its relation with $N u_{c}$ and $N u_{r}$.

The effect of optical thickness on $N u_{c}$ and $N u_{t}$ are shown in Figure 4. It is seen from Figure 4(a) that the effect of $\tau$ on $N u_{c}$ is similar to that of RC parameter (Figure 3). But if one focuses on Figure 4(b) in which the effect of $\tau$ on $N u_{t}$ is presented, it can be found that by increasing in $\tau$ from 0.1 to 0.5 (optically thin media), $N u_{t}$ has increasing trend. But with more increase in $\tau$ from 0.5 to higher values, the trend has been reversed. This is the reason why the curve for $\tau=1$ lies between the curves for $\tau=0.1$ and $\tau=0.5$. It should be noted that similar results have been reported by Tsai and ozisic [14].

\section{Conclusion}

Numerical simulation of 2-D turbulent forced convection in a duct with a BFS has been studied, including thermal radiation. The effects of RC parameter and optical thickness on the Nusselt numbers distribution along with the bottom wall downstream of the channel step were presented. Numerical results show that by increasing in RC parameter, the $N u_{c}$ decreases whereas the $N u_{t}$ increases along the bottom wall. Also, numerical results revealed that by increasing the optical thickness, the $N u_{c}$ decreases

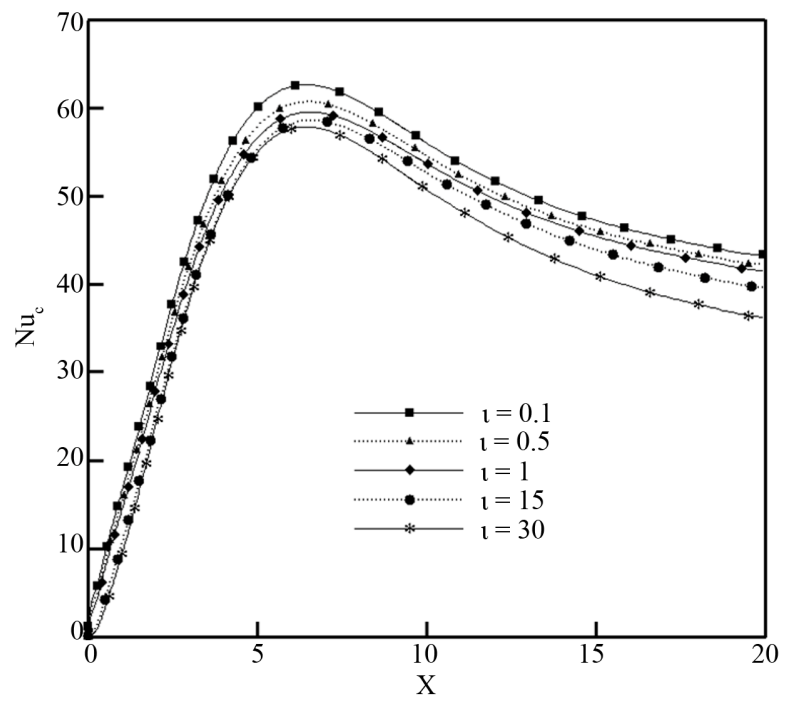

(a)

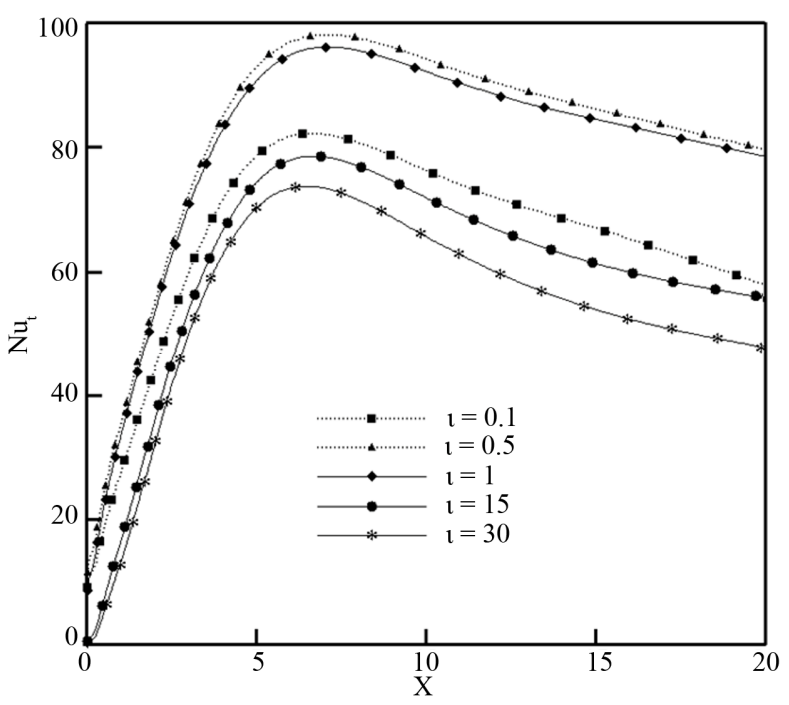

(b)

Figure 4. Effect of optical thickness on the Nusselt number distribution along the bottom wall, $\mathrm{RC}=25, \omega=0.5$ : (a) Convective Nusselt number; (b) Total Nusselt number.

monotonically, but the $N u_{t}$ increases to a critical value for the optical thickness. Such that by increasing the optical thickness from $\tau_{\text {critical }}$ to greater values, the $N u_{t}$ has a decreasing trend.

\section{REFERENCES}

[1] B. F. Armaly, F. Durst, J. C. F. Pereira and B. Schnung, "Experimental and Theoretical Investigation of Backward-Facing Step Flow,” Journal of Fluid Mechanics, Vol. 127, 1983, pp. 473-496. doi:10.1017/S0022112083002839

[2] B. F. Armaly, A. Li and J. H. Nie, "Measurements in Three-Dimensional Separated Flow,” International Jour- 
nal of Heat Mass Transfer, Vol. 46, No. 19, 2003, pp. 3573-3582. doi:10.1016/S0017-9310(03)00153-4

[3] E. W. Adams, J. P. Johnston and J. K. Eaton, "Experiments on the Structure of Turbulent Reattaching Flow," Technical Report MD-43, Thermo-Sciences Division, Department of Mechanical Engineering, Stanford University, Stanford, 1984.

[4] J. C. Vogel and J. K. Eaton, "Combined Heat Transfer and Fluid Dynamic Measurements Downstream of a Backward-Facing Step," ASME Journal of Heat Transfer, Vol. 107, No. 4, 1985, pp. 922-929. doi:10.1115/1.3247522

[5] K. Abe, T. Kondoh, and Y. Nagano, "A New Turbulence Model for Predicting Fluid Flow and Heat Transfer in Separating and Reattaching Flows-I. Flow Field Calculations," International Journal of Heat Mass Transfer, Vol. 37, No. 1, 1994, pp. 139-151. doi:10.1016/0017-9310(94)90168-6

[6] K. Abe, T. Kondoh and Y. Nagano, "A New Turbulence Model for Predicting Fluid Flow and Heat Transfer in Separating and Reattaching Flows-II. Thermal Field Calculations," International Journal of Heat Mass Transfer, Vol. 38, No. 8, 1995, pp. 1467-1481. doi:10.1016/0017-9310(94)00252-Q

[7] H. Bouali and A. Mezrhab, "Combined Radiative and Convective Heat Transfer in a Divided Channel," International Journal of Numerical Methods for Heat and Fluid Flow, Vol. 16, No. 1, 2006, pp. 84-106. doi:10.1108/09615530610636973

[8] D. G. Barhaghi and L. Davidson, "Large-Eddy Simulation of Mixed Convection-Radiation Heat Transfer in a Vertical Channel," International Journal of Heat and
Mass Transfer, Vol. 52, No. 17-18, 2009, pp. 3918-3928. doi:10.1016/j.ijheatmasstransfer.2009.03.031

[9] M. F. Modest, "Radiative Heat Transfer," Academic Press, San Diego, 2003.

[10] A. B. Ansari and S. A. Gandjalikhan Nassab, "Study of Laminar Forced Convection of Radiating Gas over an Inclined Backward Facing Step under Bleeding Condition Using the Blocked-Off Method," ASME Journal of Heat Transfer, Vol. 133, No. 7, 2011, pp. 3573-3582. doi:10.1115/1.4003607

[11] M. S. Ko, "Numerical Simulation of Three-Dimensional Combined Convective Heat Transfer in Rectangular Channels,” Ph.D. Dissertation, Texas A\&M University, College Station, 2007.

[12] S. V. Patankar and D. B. Spalding, "A Calculation Procedure for Heat, Mass and Momentum Transfer in ThreeDimensional Parabolic Flows,” International Journal of Heat Mass Transfer, Vol. 15, No. 10, 1972, pp. 17871806. doi:10.1016/0017-9310(72)90054-3

[13] Y. T. Chen, J. H. Nie, B. F. Armaly and H. T. Hsieh, "Turbulent Separated Convection Flow Adjacent to Backward-Facing Step-Effects of Step Height,” International Journal of Heat and Mass Transfer, Vol. 49, No. 19-20, 2006, pp. 3670-3680. doi:10.1016/j.ijheatmasstransfer.2006.02.024

[14] J. R. Tsai and M. N. Ozisik, "Radiation and Laminar Forced Convection of Non-Newtonian Fluid in a Circular Tube," International Journal of Heat and Fluid Flow, Vol. 10, No. 4, 1989, pp. 361-365. doi:10.1016/0142-727X(89)90027-1 


\section{Nomenclature}

ER: expansion ratio, $H /(H-h)$

I: radiation intensity

$I_{b}$ : black body radiation intensity

$k$ : turbulent kinetic energy, $\overline{u_{i}^{\prime} u_{i}^{\prime}} / 2$

$p$ : pressure

$P e$ : Peclet number, $R e \cdot P r$

$P r, P r_{t}$ : molecular and turbulent Prandtl number

$\boldsymbol{q}_{r}$ : radiative heat flux vector

$R e$ : Reynolds number, $u_{0} h / v$

RC: radiation-conduction parameter, $\sigma T_{w}^{3} h / \kappa$

St: Stanton number, $\frac{q_{w}}{\rho C_{p} u_{0}\left(T_{w}-T_{0}\right)}$

$T, t$ : mean temperature and temperature fluctuation

$\hat{t}$ : time

$u_{i}$ : general notation for mean velocity components

$\left(u_{1}=u, u_{2}=v\right)$

$u^{\prime}$ : velocity fluctuation

$x_{i}$ : general notation for coordinate directions

$\left(x_{1}=x, x_{2}=y\right)$

$y_{n}$ : normal distance to the wall surface

\section{Greek Symbols}

$\alpha, \alpha_{t}$ : molecular and eddy diffusivity

$\beta$ : extinction coefficient $\delta_{i j}:$ Kronecker delta

$\varepsilon$ : dissipation rate of turbulent kinetic energy,

$v \overline{\left(\partial u_{i}^{\prime} / \partial x_{j}\right)\left(\partial u_{i}^{\prime} / \partial x_{j}\right)}$

$\varepsilon_{t}$ : dissipation rate of $\overline{t^{2}} / 2, \alpha \overline{\left(\partial t / \partial x_{j}\right)\left(\partial t / \partial x_{j}\right)}$

$\varepsilon_{w}$ : wall emissivity

$\kappa$ : thermal conductivity

$\Omega$ : solid angle

$v, v_{t}$ : molecular kinematic and eddy viscosities

$\sigma$ : Stefan Boltsman's constant, $5.67 \times 10^{-8} \mathrm{~W} / \mathrm{m}^{2} \cdot \mathrm{K}^{4}$

$\sigma_{s}$ : scattering coefficient

$\sigma_{a}:$ absorption coefficient

$\omega$ : albedo coefficient, $\omega=1-\sigma_{a} / \beta$

$\tau$ : optical thickness, $\beta h$

$\theta_{1}, \theta_{2}$ : dimensionless temperature parameters

$\left(\theta_{1}=T_{0} /\left(T_{w}-T_{0}\right), \theta_{2}=T_{w} / T_{0}\right)$

\section{Subscripts}

b: bulk

c: convective

r: radiative

w: wall

\section{Superscript}

*: dimensionless symbol 